\title{
Spinal myoclonus
}

\author{
A. P. HOPKINS AND W. F. MICHAEL
}

From the Department of Neurology, St Bartholomew's Hospital, London

SYNOPSIS A case of rhythmic myoclonus affecting only the lower part of the body is described. This occurred as an acute self-limiting illness. The changes in the cerebrospinal fluid (CSF) suggested a viral infection. Clinical and electrophysiological findings indicated that the involuntary movements were arising at spinal level and were independent of suprasegmental influences. There are few previously reported cases of spinal myoclonus, all different in various respects from the present one, which is reminiscent of the results of experimental inoculation of virus into feline spinal cord.

Myoclonus may be defined as a sudden jerk which occurs in a muscle or in part of a muscle, and which is not under voluntary control. Myoclonic jerks may be caused by lesions at various levels of the nervous system, but evidence that clearly shows that myoclonus arises at the spinal level is sparse. We present a case of acute spinal myoclonus with virtually complete recovery. Electrophysiological studies helped confirm that these movements were of spinal origin.

\section{CASE REPORT}

(St.B.H. 510289) A previously healthy barrister aged 36 years returned to England from a holiday in Greece. A few hours after his return he noticed a feeling of heaviness of the legs and difficulty in walking. Shortly after, his legs began to twitch, and he noticed some hesitancy of micturition. He attended a casualty department, where a diagnosis of hysteria was made and he was sent home. The twitching of his legs became continuous and more violent, and he was unable to sleep at all that night, although he did not feel ill. He was admitted next morning to St Bartholomew's Hospital.

On examination, he was a fit-looking man, without fever or neck stiffness. The cranial nerves and arms were normal. There were continuous rhythmic thrusting movements of the pelvis and repetitive jerks of the legs. These sometimes were bilaterally synchronous. Their frequency was about 100 to 150 times per minute, and their amplitude sufficiently violent to shake the bed. Because of the transmitted movement it was difficult to be sure of the upper limit of muscles involved by these movements, but it appeared that all muscles above segmental level T12 were spared. Full voluntary power of the legs was retained. The jerks were not modified by voluntary movement, and they continued unabated during $\vec{\circ}$ sleep. Examination of other systems was normal.

He was treated with diazepam $30 \mathrm{mg}$ a day. Afte four days the jerks were less violent and no longe $N$ continuous. He was able to walk without obvious
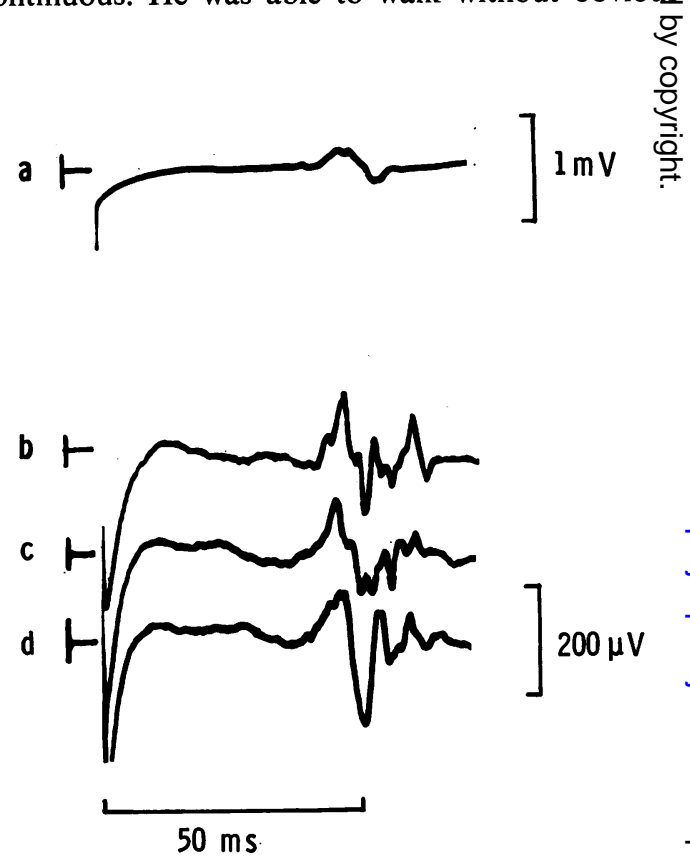

FIG. 1. Muscle action potentials recorded from left gastrocnemius evoked by stimulating the left medial $\mathrm{S}$ popliteal nerve (a). The evoked responses from left $N$ gastrocnemius on constant stimulation of the right $\mathrm{N}$ medial popliteal nerve are shown in $\mathrm{b}, \mathrm{c}$, and $\mathrm{d}$. 
myoclonus and he could lie in bed without jerks for increasingly long periods. He could, however, still bring on bouts of myoclonus by adopting certain postures, particularly by turning on to his side with legs and knees flexed. Myoclonus was also precipitated by vibration induced by tapping the clavicle, or even by tapping the bed. Loud sounds had no effect. Tapping the tendon of a muscle of the lower limb elicited a contraction not only in that muscle, but in other muscles of both lower limbs. Tapping the Achilles tendon resulted in a simultaneous contraction of both tibialis anterior and triceps surae, but the net result was always dorsiflexion of the foot.

INVESTIGATIONS The haemoglobin concentration, white cell count, and differential count were normal. The ESR was $23 \mathrm{~mm}$ in the first hour, and radiographs of the chest were negative. Four days after the first symptoms the cerebrospinal fluid (CSF), under normal pressure, contained 57 lymphocytes and 5 polymorphs per $\mathrm{mm}^{3}$; protein $107 \mathrm{mg} / 100 \mathrm{ml}$, IgG $7.0 \mathrm{mg} / 100 \mathrm{ml}$; sugar $69 \mathrm{mg} / 100 \mathrm{ml}$. Bacterial culture was sterile. The CSF was examined again on the ninth day of the illness. On this occasion there were 58 red cells, 40 lymphocytes, and 3 polymorphs per $\mathrm{mm}^{3}$. The protein had fallen to $56 \mathrm{mg} / 100 \mathrm{ml}, \mathrm{IgG}$ $6.6 \mathrm{mg}$. On the sixth day, complement fixing antibodies to Herpes simplex $(1: 256)$, H. zoster $(1: 1024)$, and measles $(1: 64)$ were found in the blood, but no

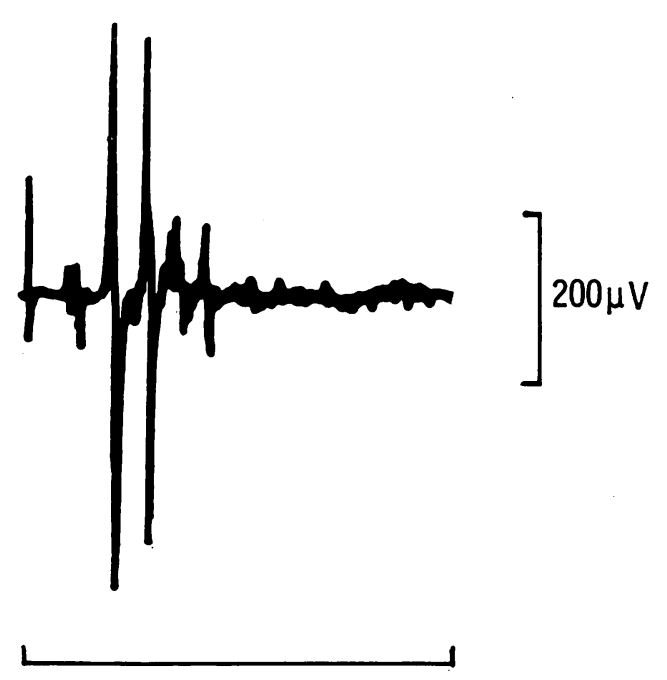

$500 \mathrm{~ms}$

FIG. 2. Repetitive firing in left gastrocnemius in response to a single shock to the right medial popliteal nerve.

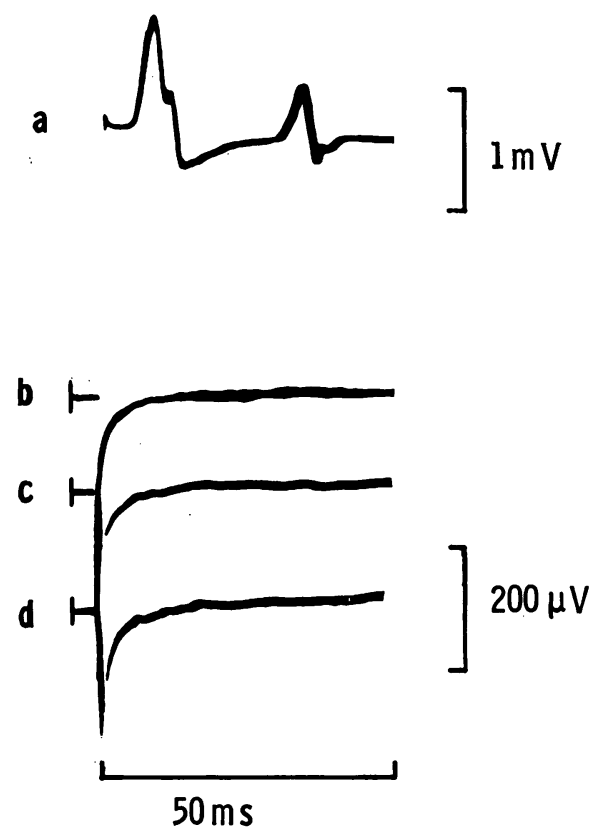

FIG. 3. (a) A normal Hoffmann response in left gastrocnemius after clinical recovery. In $\mathrm{b}, \mathrm{c}$, and $\mathrm{d}$ note absence of response on stimulation of contralateral medial popliteal nerve.

antibodies to these viruses were present in the CSF. Enteroviruses were not isolated from monkey kidney tissue culture inoculated with stools and CSF. Stools were inoculated into suckling mice in a search for Coxsackie virus, with negative results.

Electrophysiological studies were performed five days after the onset of the illness, at a time when the jerks were no longer continuous. Stimulating cathodes were placed over both medial popliteal nerves, the anodes being over the patellae. Surface recording electrodes were placed over the left gastrocnemius muscle. At a stimulus intensity insufficient to cause a direct (M) response in the left gastrocnemius muscle, widespread myoclonic jerks in both lower limbs appeared in response to stimulating the left medial popliteal nerve. These jerks continued for 1-2 s. The initial negative deflection shown in Fig. 1a had a latency of about $43 \mathrm{~ms}$. When the right popliteal nerve was stimulated at an intensity insufficient to cause a twitch of the right gastrocnemius at intervals of about $10 \mathrm{~s}$, the initial negative response from left gastrocnemius also had a latency of about $40 \mathrm{~ms}$. The shape and amplitude of the potential evoked in the left gastrocnemius varied in shape and amplitude (Fig. 1b, c, d), even though the stimulus was con- 
stant. On a different sweep speed (Fig. 2) repetitive synchronous firing of many motor units can be seen at intervals of $40-50 \mathrm{~ms}$. Examination of the left gastrocnemius and left tibialis anterior at rest with a concentric needle electrode showed occasional fasciculation potentials, but was otherwise normal. When seen three weeks after the onset of the illness, no myoclonus could be induced, and both knee and ankle tendon jerks were absent, although the arm tendon jerks remained normal. The left ankle jerk had returned two weeks after this. At this time an apparently normal $\mathrm{H}$ reflex with a latency of $30 \mathrm{~ms}$ could be obtained from the left gastrocnemius muscle on stimulating the left medial popliteal nerve (Fig. 3a) but there was no response in the left gastrocnemius to stimulation of the right medial popliteal nerve (Fig. 3b). Occasional fasciculation potentials were found in the left gastrocnemius.

\section{DISCUSSION}

An early demonstration that involuntary movements might arise in the spinal cord was that of Turtschaninow (1894), who produced jerks in the legs of dogs by intravenous injection of $5 \%$ phenol. Since the jerks were not abolished by transection of the brain-stem or spinal cord although eliminated by section of the peripheral nerves, they were clearly arising in the spinal cord. Other relevant animal experiments are those of Luttrell et al. (1959), who injected the virus of Newcastle disease into the spinal cord of cats. Bilateral synchronous jerks developed in the muscles supplied by the injected segments, and remained unaffected by surgical isolation of these segments.

The possibility of a viral infection was considered by Campbell and Garland (1956) who reported three patients who suffered a rapidly fatal illness, characterized by myoclonic jerks of the lower limbs. Histological changes strongly suggestive of a viral infection were found, almost exclusively confined to the spinal cord. The main changes were lymphocytic cuffing and microglial nodules. These authors also mention that damage to the anterior horn cells was inconspicuous. Their cases differ from the present one in that the patients were all extremely ill, with fever, sweating, and severe pain from the muscular spasms. The general condition of our patient never gave cause for alarm, and pain was not a feature. Two of their patients had received recent radiotherapy to the spine, and they speculated on the role this might have played in predisposing to viral infections. Activation of a latent virus by irradiation would now also be 0 considered a possibility.

Other cases of spinal myoclonus described in the literature also bear little resemblance to the present case. Silfverskiöld (1962) collected three cases all of which began in the autumn of 1958 in the area of Stockholm, suggesting infection as the causal agent, but no such agent could be demonstrated. All were children who had rhythmic jerks of some muscles of arms and legs which did not interfere with voluntary action. These movements persisted for up to 18 months. Electromyography in one case demonstrated that brief jerks affected different muscles in the same limb synchronously, though they were seldom simultaneous in two extremities. However, it appears that the involuntary movements were not entirely independent of supraspinal control as they were increased by emotion and decreased both during voluntary use of the limb and during $\vec{\nabla} \vec{N}$ mental arithmetic; they also disappeared durin ${ }^{\circ}$ 을 sleep. Swanson et al. (1962) reported two patients with localized involuntary movements considered to be myoclonic, of a benign nature in both; one had lifelong jerks of the left arm and $\stackrel{\mathbb{D}}{-}$ abdominal muscles, the other had a 10 mont $\overrightarrow{0}$ history of twitching of the muscles of the left leg. In both cases, these movements were no more than a nuisance. Electromyography showed brief asynchronous discharges, sometimes only in parts of individual muscles.

A convincing case of myoclonus of purely spinal origin was described by Garcin et al. (1968). Myoclonus of the right upper arm was produced by an astrocytoma of the spinal cord, extending between the $\mathrm{C} 3$ and $\mathrm{C} 5$ vertebral bodies. The frequency of jerks was 43 per minute, and they were synchronous in all the muscles affected. The amplitude was only slightly modified by passive movement of the limb, but voluntary contraction of any of the affected muscles temporarily abolished its myoclonus. Passive stretch of the muscle had no effect. The myoclonus was continuous during sleep. After surgical removal of the tumour, the jerks changed in character and were replaced by irregular asynchronous contractions of biceps and supinator longus.

In the present case, there was good evidence 
for purely spinal disease. The jerks were confined to the lower part of the trunk and legs. Many muscles were affected synchronously and fairly symmetrically between the two sides. The movements continued during sleep, and they were not exacerbated by loud noises or voluntary movements. When spontaneous myoclonus had ceased, manoeuvres which directly or indirectly stretched the muscle spindles, such as tapping tendons or the skeleton, or knocking the bed, were effective in eliciting myoclonic jerks. Furthermore, it was demonstrated that stimulation of the medial popliteal nerve induced myoclonic jerks in both ipsilateral and contralateral legs with almost identical latencies of about $40 \mathrm{~ms}$. This is a little longer than the $37 \mathrm{~ms}$ found by Marsden et al. (1973) for the latency of the ankle jerk in a normal subject, but considerably shorter than the $75 \mathrm{~ms}$ latency they found for the tonic stretch reflex in the long flexor of the big toz, which they suggest involves a loop via fast conducting fibres through the cerebral cortex. Mayer and Mawdsley (1965) reported a mean latency of $29 \mathrm{~ms}$ for the $\mathrm{H}$ reflex recorded from the mid-calf in young adults (range $26-32 \mathrm{~ms}$ ). The latency of the myoclonic jerks in our patient induced by stimulation of the medial popliteal nerve thus suggests conduction through a spinal pathway with more than one synapse, the figure of $40 \mathrm{~ms}$ being too long for a monosynaptic route, and probably too short for any pathway extending up to supraspinal levels. The absence of clinical weakness and of denervation poten- tials in the present case, and the subsequent disappearance of the tendon reflexes in the affected segments suggests that the predominant lesion lay in the intercalated neurones of the dorsal horn, the anterior horn cells remaining largely intact.

We thank Dr A. M. Halliday for his comments on the manuscript. A.P.H. thanks the British Epilepsy Association for support. Apparatus was provided by the Joint Research Board of St Bartholomew's Hospital.

\section{REFERENCES}

Campbell, A. M. G., and Garland, H. (1956). Subacute myoclonic spinal neuronitis. Journal of Neurology, Neurosurgery, and Psychiatry, 19, 268-274.

Garcin, R., Rondot, P., and Guiot, G. (1968). Rhythmic myoclonus of the right arm as the presenting symptom of a cervical cord tumour. Brain, 91, 75-84.

Luttrell, C. N., Bang, F. B., and Luxenberg, K. (1959). Newcastle disease encephalomyelitis in cats. 2. Physiological studies on rhythmic myoclonus. Archives of Neurology, 81, 285-291.

Marsden, C. D., Merton, P. A., and Morton, H. B. (1973). Is the human stretch reflex cortical rather than spinal? Lancet, 1, 759-761.

Mayer, R. F., and Mawdsley, C. (1965). Studies in man and cat of the significance of the $\mathrm{H}$ wave. Journal of Neurology, Neurosurgery, and Psychiatry, 28, 201-211.

Silfverskiöld, B. P. (1962). Rhythmic myoclonus in three girls. Acta Neurologica Scandinavica, 38, 45-59.

Swanson, P. D., Luttrell, C. N., and Magladery, J. W. (1962.) Myoclonus-A report of 67 cases and review of the literature. Medicine (Baltimore), 41, 339-356.

Turtschaninow, P. (1894). Experimentelle Studien über den Ursprungsort einiger klinisch wichtiger toxischer Krampfformen. Archiv für Experimentelle Pathologie und Pharmakologie, 34, 208-246. 